\title{
TILs and Anti-PD1 Therapy: An Alternative Combination Therapy for PDL1 Negative Metastatic Cervical Cancer
}

\author{
Huanhuan Yin, Wei Guo, Xiangling Sun, Ruili Li, Cuihua Feng, and Yujie Tan \\ Department of Obstetrics, Luoyang Central Hospital Affiliated to Zhengzhou University, Luoyang 471009, China \\ Correspondence should be addressed to Yujie Tan; zq.7800@163.com
}

Received 30 June 2020; Revised 23 August 2020; Accepted 27 August 2020; Published 7 September 2020

Academic Editor: Nejat K. Egilmez

Copyright ( $) 2020$ Huanhuan Yin et al. This is an open access article distributed under the Creative Commons Attribution License, which permits unrestricted use, distribution, and reproduction in any medium, provided the original work is properly cited.

\begin{abstract}
Background. We investigated the efficacy of TILs and anti-PD1 combination therapy in patients with metastatic cervical cancer with low MSI expression and PDL1-negative. Methods. A total of 80 patients were put on TILs and anti-PD1 combination therapy, and the progression-free survival time (PFS) and overall survival time (OS) were assessed by Kaplan-Meier analysis. Univariate and multivariate analyses were performed to identify factors that could predict the prognosis of metastatic cervical cancer in the previously described patients. Results. The objective response rate was $25 \%$, whereas the mPFS and mOS were 6.1 and 11.3 months, respectively. The therapeutic efficacy was influenced by the characteristics of TILs, infection with HPV, and development of fever just after the therapy. Conclusion. Overall, our results show that the combination therapy of TILs and antiPD1 significantly improves the prognosis of metastatic cervical cancer.
\end{abstract}

\section{Introduction}

Globally, cervical cancer is not only one of the major health complications affecting women but also the fourth most prevalent cancer in women [1].Epidemiologically, $85 \%$ of all cancer cases occur in developing countries, where cervical cancer leads in the number of cancer-related mortality among women [2]. In 2019 alone, 13170 new cases of uterine cervical carcinomas were reported in the united states, where 4250 people died of the disease [3]. Although the prevalence of cervical cancer is generally decreasing among women in the United States, the incidence rates remain high among Hispanic/Latino, Black, and Asian women [4-7]. The primary treatment for early-stage cervical cancer is either surgery or radiation therapy. On a positive note, the 5-year progressionfree survival rate (PFS) of patients with cervical cancer exceeds $70 \%$ [8]. However, metastatic types or recurrent lesions are not amenable to radical local excision or regional radiation and generally have a poor prognosis. Consequently, they are treated with the more toxic palliative platinum-based chemotherapy. Median overall survival (OS) rates range from 6.5 to 13.3 months for chemotherapy regimens alone, and 6.5 to 16.8 months for bevacizumab-containing regimens [9-12]. Unfor- tunately, there are few effective therapeutic options for patients with recurrent tumors which are resistant to firstline chemotherapy, and thus, this group of patients exhibit poor prognosis to the available regimens. Therefore, metastatic cervical cancer presents one of the biggest challenges in clinical practice. In view of this, there is an urgent need to develop new therapeutic options, effective against metastatic cervical cancer.

Under normal physiological conditions, immune checkpoints play a crucial role in the prevention of autoimmunity [13]. To survive in the body, cancer cells express programmed death-ligand 1 (PD-L1), a molecule that can modulate the immune checkpoints and thus downregulate the generation of tumor-specific T cells. In effect, cancer cells protect themselves against immune attack [14]. Immune checkpoint inhibitors reduce the interaction between cancer cells and activated $\mathrm{T}$ cells which destroy malignant targets. Immunotherapy with immune checkpoint inhibitors has emerged as a novel treatment for patients with advanced cancers. Recent evidence has demonstrated the potential efficacy of immune checkpoint inhibitors in cervical cancer [15]. The objective response rate (ORR) for these treatments in recurrent and/or advanced cervical cancer ranges from 12.2 to $26 \%$ [16]. However, the 
selection of patients that potentially responds positively to this therapy remains challenging. Increased expression of PD-L1 on tumor and immune cells and high levels of microsatellite instability (MSI-high) have been associated with better response to immunotherapies [17]. However, the response rate of metastatic cervical cancer in patients with PDL1negative and low MSI expression is relatively poor when put on anti-PD1 therapy. Therefore, anti-PD1 therapy alone may not be an effective treatment method for metastatic cervical cancers, particularly in patients testing negative for PDL1 and low MSI expression.

Adoptive T cell therapy (ACT) is an emerging cancer treatment modality that involves systemic infusion of therapeutic $\mathrm{T}$ cells. It has been shown to induce complete tumor responses in some patients with B cell malignancies or metastatic melanoma [18]. In one phase II study on tumorinfiltrating lymphocyte (TIL) therapy for the treatment of patients with metastatic carcinomas associated with human papillomavirus (HPV), it was found that TILs yielded an objective response rate (ORR) of $28 \%(5 / 18)$ in patients with cervical cancer [19]. Interestingly, the reactivity of HPV to the infused $\mathrm{T}$ cells together with repopulation of peripheral blood with HPV-reactive T cells positively correlated with better clinical response [20]. These findings support the concept that TILs therapy can inhibit metastatic cervical cancer. The effects of anti-PD1 therapy are mediated by TILs in the tumor microenvironment; therefore, a combination of antiPD1 therapy and TILs may confer superior antitumor effects against metastatic cervical cancer than current therapies.

This study was primarily aimed at assessing the response to a combination of adoptive TILs and anti-PD1 in patients with chemotherapy-resistant cervical cancer. We also sought to determine potential biomarkers associated with better response to TILs and anti-PD1 combination therapy. This will help in identifying patients most likely to respond better to the newly proposed therapy.

\section{Materials and Methods}

2.1. Study Design. This single-center clinical study was approved by the Ethics Committee at the Affiliated Luoyang Central Hospital of Zhengzhou University. All methods and procedures associated with this study were conducted in accordance with the Good Clinical Practice guidelines and accorded ethically with the principles of the Declaration of Helsinki and local laws. All authors had access to the study data and reviewed and approved the final manuscript. Infusions of anti-PD1 therapy (nivolumab, $3 \mathrm{mg} / \mathrm{kg}$ ) were administered to the patients at our department two weeks for one cycle. All patients received at least 8 cycles of infusions or received cycles until they experienced disease progression or unacceptable adverse effects (AEs) or withdrew this study. At the first cycles of anti-PD1 therapy, TILs were transfused into patients. Patients with disease progression received multidisciplinary synthetic therapy and best support cares. After treatment, all the patients received follow-up to examine the tumor status every 3 months. The follow-up deadline was June 20, 2020.
2.2. Patients Selection. Eighty patients with a clinical diagnosis of cervical cancer were enrolled in this study. In addition, the study group had experienced disease progression after first-line chemotherapy. Other inclusion criteria included the following: (1) discontinuing any cancer therapy before enrollment, (2) be an aged above 35 years old, (3) life expectancy of more than 3 months, (4) Eastern Cooperative Oncology Group (ECOG) performance status of $0-1$, (5) adequate organ function, and (6) lesions that can be assessed using the standard response evaluation criteria in solid tumors (RECIST 1.0 version 1.1) guidelines [21]. The following exclusion criteria were applied: previous treatment with anti-CTLA4 or anti-PD1/PDL1 therapy, any form of primary immunodeficiency or history of autoimmune diseases, ongoing systemic infections, and concurrent systemic steroid therapy, and recruitment into other clinical trials. All participating patients provided informed consent.

2.3. Outcome Measures. The primary endpoint for this research was to evaluate the safety and AEs associated with a combination therapy of TILs and nivolumab in patients with cervical cancer. Secondary endpoints included the assessments of the objective response rate (ORR), progression-free survival time (PFS), and overall survival time (OS). Safety evaluations primarily consisted of any abnormalities identified clinically or in the laboratory, arising at any stage of the study up until two weeks after the last infusion of nivolumab. AEs were evaluated based on the guidelines set by the National Cancer Institute (NCI) on Common Toxicity Criteria (CTC), version 4.0 [22]. Therapy-associated AEs were assessed during the treatment and observation periods, where the most severe events for each patient based on the grading scores were also recorded. The cancer lesions were evaluated using computed tomography (CT) or magnetic resonance imaging (MRI) after every 3 months. The ORR was assessed using RECIST version 1.1 [21]. The ORR was defined as complete response (CR) plus partial response $(\mathrm{PR})$, and disease control rate (DCR) was defined as CR plus PR plus stable disease (SD). Potential prognostic factors were also evaluated using various univariate and multivariate analyses. The PFS was calculated from the date a patient was put on the immunotherapy to the time the disease worsens. Patients who did not show any improvement were censored at the time of last contact. The OS was calculated from the date the first immunotherapy was administered to the time of death, and patients who were alive at the time of last contact were also censored.

2.4. Generation of TILs. TILs were obtained by culturing fresh tumor tissues from metastatic sites, extracted from each patient using a thick needle puncture. The tumor tissues were evaluated by two independent pathologists before the cultures were made. The experiment was performed as previously described $[19,23,24]$. Briefly, tumor tissues were sliced into small pieces of about 2 to $3 \mathrm{~mm}^{3}$ using a scalpel. Cell suspensions were made by digesting the tissues using collagenase type IV (Sigma-Aldrich, St. Louis, MO, USA, $1 \mathrm{mg} / \mathrm{mL}$ ), DNase I (Sigma-Aldrich, St. Louis, MO, USA, $2 \mathrm{U} / \mathrm{mL}$ ), and hyaluronidase type V (Sigma-Aldrich, St. Louis, MO, USA, 
$0.5 \mathrm{U} / \mathrm{mL}$ ) for approximately 3 hours at room temperature. The single-cell suspensions were filtered, washed twice with phosphate-buffered saline (PBS), and incubated in a 12-well plate at a concentration of $1.0 \times 10^{6} \mathrm{TILs} / \mathrm{mL}$ in an XVIVO medium (Muenchensteinerstrasse $38 \mathrm{CH}-4002$ Basel, Switzerland) containing $7000 \mathrm{IU} / \mathrm{mL}$ of recombinant human interleukin-2 (rhIL-2, Novartis, UK). The day of culturing was considered as day 0 . The cell suspensions were removed and further purified using Ficoll gradient at day 1 . The purified bulk of TIL were recultured in X-VIVO medium mixed with $7000 \mathrm{IU} / \mathrm{mL}$ rhIL-2 and maintained at a concentration of $1-2 \times 10^{6}$ cells $/ \mathrm{mL}$ until all other cells (including cancer cells) were eliminated. This was done to achieve a concentration of at least $5 \times 10^{7} \mathrm{TIL}$ cells $/ \mathrm{ml}$. In general, the culturing process was performed for approximately 10 to 14 days. The cultured TIL cells were immediately incubated with anti-CD3 antibody (GE Healthcare Biosciences, Pittsburgh, PA, USA; $30 \mathrm{ng} / \mathrm{mL}$ ) mixed with $1000 \mathrm{IU} / \mathrm{mL}$ rhIL-2 for large-scale production of TIL. In the end, about $5 \times 10^{9}$ TIL cells were harvested, purified, immunophenotyped, and infused back into patients.

2.5. TILs Immunophenotyping. Immunophenotyping of TILs was performed as previously described $[23,24]$. The postculture phenotypes of TILs were characterized using flow cytometry. The cells were first labeled using anti-CD3-FITC (Cat\#: 555339, $1.5 \mu \mathrm{l} / 10^{6}$ cells), anti-CD4-APC-Cy7 (Cat\#: 557871, $2 \mu \mathrm{l} / 10^{6}$ cells), anti-CD8-BV786 (Cat\#: 563823, $2 \mu \mathrm{l} / 10^{6}$ cells), anti-CD56-BV421 (Cat\#: 562752, $3 \mu \mathrm{l} / 10^{6}$ cells), and anti-PD1-PE-Cy7 (Cat\#: $561272,5 \mu 1 / 10^{6}$ cells) after a 30 minutes culture on ice and darkness. Thereafter, the cells were washed once with PBS and resuspended in $400 \mu \mathrm{l}$ PBS. Live and dead cells were distinguished using $7 \mathrm{ADD}$. They were then run on a BD Fortessa (BD Bioscience). Negative control of each channels was labeled using Fluorescence minus one (FMO) technique as previously described [25]. The data generated in the flow cytometry was analyzed using the FlowJo software. To analyze T regulatory cells, FoxP3 staining was performed using an intracellular staining protocol following manufacturers' protocol. Next, anti-CD3 and anti-CD4 were first stained for 30 minutes on ice in the dark. TILs were washed, fixed with the labeled anti-CD3 and anti-CD4, and permeabilized using BD Fix Buffer I (Cat\#: 557870, BD bioscience, USA) and Perm Buffer III (Cat\#: 558050, BD bioscience, USA) following the manufacturers' protocol. The cells were washed thrice with Perm Buffer III and incubated for 30 minutes with antiFoxP3-V450 (Cat\#: 560460, $5 \mu \mathrm{l} / 10^{6}$ cells) under ice and in darkness. The cells were then run on a BD Fortessa (BD Bioscience). Fluorescence minus one (FMO) staining was used to generate negative controls. The data generated by flow cytometry was analyzed using the Flowjo software. The gating strategies for T cell phenotype were as follows: (1) plot of FSC-A and SSC-A; (2) plot of FSC-A and FSC-H to gate single cells; (3) plot of 7AAD negative populations to gate live cells. Based on these gating, we then gated $\mathrm{CD}^{+} \mathrm{T}$ cell populations. For $\mathrm{CD}^{+} \mathrm{CD}^{+} \mathrm{T}$ cells or $\mathrm{CD}^{+}{ }^{+} \mathrm{CD} 8^{+} \mathrm{T}$ cells or $\mathrm{CD}^{+}{ }^{+} \mathrm{CD} 56^{+} \mathrm{NKT}$ cells, we gated $\mathrm{CD} 4^{+}$or $\mathrm{CD} 8^{+}$or $\mathrm{CD} 56^{+}$ populations followed by $\mathrm{CD}^{+}$populations. For NK cells populations, we gated $\mathrm{CD}^{-}$populations and then gated CD56 $6^{+}$populations. To determine the percentage of $\mathrm{PD}^{+}$ cells on $\mathrm{CD} 3^{+} \mathrm{CD}^{+} \mathrm{T}$ cells, we firstly gated $\mathrm{CD} 3^{+} \mathrm{T}$ cells, then gated $\mathrm{CD}^{+} \mathrm{T}$ cells followed by $\mathrm{CD} 3^{+}$populations. Finally, we gated $\mathrm{PD} 1^{+}$populations followed by $\mathrm{CD} 3^{+} \mathrm{CD} 8^{+}$populations. For Treg cells population, we first gated $\mathrm{CD} 3^{+} \mathrm{CD} 4^{+} \mathrm{T}$ cells population and then gated FoxP $3^{+}$population.

2.6. Cytotoxicity and Cytokines Production of TILs. Antitumor activity of TILs was assessed using an overnight cytotoxicity assay. Here, Hela cell line was used as target. Cytotoxic activity was determined using a lactate dehydrogenase (LDH) release assay as previously described. This nonradioactive assay is a colorimetric alternative to the ${ }^{51} \mathrm{Cr}$ release assay that quantitatively measures LDH released upon cell lysis. In each assay, effector cell concentration at $40: 1$, $20: 1$, and $10: 1$ was evaluated. For the cytokines production of TILs, we measured the release of IFN- $\gamma$ (BV650-conjugated, Cat\#: 563416), IL-2 (BV510-conjugated, Cat\#: 564167), and TNF- $\alpha$ (APC-conjugated, Cat\#: 551384), using an intracellular staining protocol following manufacturers' protocol for both fresh TILs and cultured TILs. We compared the expression of IFN- $\gamma$, IL- 2 , and TNF- $\alpha$ on $\mathrm{CD}^{+} \mathrm{T}$ cells before and after culture. The intracellular staining method is the same to FoxP3 staining.

2.7. Statistical Analysis. GraphPad Prism 7.0 and Spss 17.0 software was used for statistical analysis. PFS and OS were calculated by Kaplan-Meier. OS and PFS were calculated from the start of TILs therapy. Univariable and multivariable Cox proportional hazards regression models were used to estimate hazard ratios along with associated confidence intervals and $P$ values. Other data used $t$-test or $\chi^{2}$-test. For all statistical analyses, significance is indicated as at least $P<0.05$.

\section{Results}

3.1. Patient Characteristics. Between June 2017 and March 2019, 80 patients with persistent metastatic cervical cancer after developing resistance to first-line chemotherapy were enrolled and put on a combination therapy of TILs and nivolumab. Briefly, $25 \%$ of the patients received a combination of first-line chemotherapy and bevacizumab, whereas the rest, $75 \%$ were put on chemotherapy alone. The median age of patients was 45 , with ages ranging from 35 to 65 years. The most common sites for metastatic cervical cancer were lungs and lymph nodes, which accounted for $55 \%$ and $40 \%$, respectively. HPV subtyping for strain $6,11,16,18$, or 33 was also performed for patients who tested positive for the virus. Notably, all the patients were PD-L1-negative (both tumor cells and immune cells were negative) and exhibited low expression of MSI. Detailed characteristics of the patients are shown in Table 1.

3.2. Phenotype for TILs. Each patient was infused with an average of $50 \times 10^{9}$ TILs (range, $35-88 \times 10^{9}$ ). The phenotype of TILs was examined by flow cytometry and representative dot plots for $\mathrm{T}$ cell phenotypes are shown in supplementary Figure 1. The TILs primarily comprised of $\mathrm{CD}^{+} \mathrm{T}$ cells $(93.54 \% \pm 6.23 \%, N=80), \mathrm{CD}^{+} \mathrm{T}$ cells 
TABle 1: Patient characteristics $(N=80)$.

\begin{tabular}{|c|c|c|}
\hline Characteristic & No. of patients & $\%$ \\
\hline \multicolumn{3}{|l|}{ Age (years) } \\
\hline$\geq 45$ & 45 & 56.25 \\
\hline$<45$ & 35 & 43.75 \\
\hline \multicolumn{3}{|l|}{ Pathological type } \\
\hline Squamous cell & 65 & 81.25 \\
\hline Adenocarcinoma & 15 & 18.75 \\
\hline \multicolumn{3}{|l|}{ ECOG PS } \\
\hline 0 & 50 & 62.50 \\
\hline 1 & 30 & 37.50 \\
\hline \multicolumn{3}{|l|}{ HPV status } \\
\hline Positive & 68 & 85.00 \\
\hline Negative & 12 & 15.00 \\
\hline \multicolumn{3}{|c|}{ Size of primary tumor $(\mathrm{cm})$} \\
\hline$\geq 5$ & 47 & 58.75 \\
\hline$<5$ & 33 & 41.25 \\
\hline \multicolumn{3}{|c|}{ First-line chemotherapy with bevacizumab } \\
\hline Yes & 20 & 25.00 \\
\hline No & 60 & 75.00 \\
\hline \multicolumn{3}{|c|}{ Location of metastatic tumors } \\
\hline Lung & 55 & 68.75 \\
\hline Liver & 30 & 37.50 \\
\hline Bone & 20 & 25.00 \\
\hline Lymph node & 40 & 50.00 \\
\hline Peritoneum & 35 & 43.75 \\
\hline
\end{tabular}

$(68.33 \% \pm 9.64 \%, N=80), \mathrm{CD}^{+} \mathrm{T}$ cells $(28.27 \% \pm 5.79 \%$, $N=80)$, NK cells $(3.52 \% \pm 2.96 \%, N=80)$, and NKT cells $(24.33 \% \pm 8.16 \%, N=80)$. The proportion of PD-1 was expressed as mean \pm SD of $20.34 \% \pm 8.25 \%$ of infused TILs, primarily on the $\mathrm{CD}^{+} \mathrm{T}$ cells $(17.25 \% \pm 6.28 \%, N=80)$. A subgroup of Foxp $3^{+} \mathrm{T}$ regulatory cells $(18.96 \% \pm 8.69 \%$, $N=80$ ) was measured from the $\mathrm{CD}^{+} \mathrm{CD}^{+} \mathrm{T}$ cell population.

3.3. Cytotoxicity and Cytokines Production by TILs. The antitumor effects of TILs were evaluated by measuring the cytotoxicity of TILs against Hela target cell line. TILs were cocultured with Hela cells for $72 \mathrm{~h}$ at effector to target $(\mathrm{E} / \mathrm{T})$ cell ratios of $40: 1,20: 1$, and $10: 1$. Median cytotoxicity levels of TILs were $80.78 \% \pm 5.68 \%, 55.34 \% \pm 3.76 \%$, and $38.49 \%$ $\pm 3.12 \%$ (Supplementary Figure 2A). TILs kill cancer cells by releasing cytokines, such as IFN- $\gamma$, IL-2, and TNF- $\alpha$ [26]. Flow cytometry analysis showed that cultured TILs enhanced the production of IFN- $\gamma$, IL-2, and TNF- $\alpha$ compared with fresh TILs (Supplementary Figure 2B and C). Taken together, these data showed that cultured TILs had the viability to kill tumor cells.

3.4. Treatment-Related Toxicities. The most common AEs related to the combination therapy of TILs and anti-PD-1 were fever, fatigue, rash, and anorexia (Table 2). In general, treatment-associated AEs occurred in 73 patients $(91.25 \%)$, in which grade 1 or 2 AEs were observed in $69(94.52 \%)$ of
TABLE 2: Treatment-related adverse events in patients in response to therapy $(N=80)$.

\begin{tabular}{lcc}
\hline \multirow{2}{*}{ Sides effects } & \multicolumn{2}{c}{$\begin{array}{c}\text { No. }(\%) \text { patients associated with } \\
\text { adverse events }\end{array}$} \\
& Grade 1or 2 & Grade 3 or 4 \\
\hline Fever & $50(62.50)$ & $4(5.00)$ \\
Fatigue & $18(22.50)$ & 0 \\
Rash & $16(20.00)$ & 0 \\
Anorexia & $12(15.00)$ & 0 \\
Leukopenia & $5(6.25)$ & 0 \\
Anemia & $4(5.00)$ & 0 \\
Vitiligo & 0 & 0 \\
Nausea & 0 & 0 \\
Vomiting & 0 & 0 \\
Total incidence & $69(86.25)$ & $4(5.00)$ \\
\hline
\end{tabular}

the 73 patients. Grade 3 or 4 treatment-associated AEs were observed in four patients (5.00\%). One patient exhibited a grade 4 fever during treatment, but the latter underwent objective antitumor regression (complete response, CR) after 3 months of combined TILs and nivolumab therapy. One patient who developed grade 3 fever underwent CR after 3 months of the combined therapy. Grade 3 fever was also observed in the other two patients who underwent partial recovery (PR) after 6 cycles of the combined therapy. Notably, fever was the most frequently observed $\mathrm{AE}$, which occurred in $50(62.5 \%)$ patients. However, the body temperature for nearly all cases of fever did not exceed $38^{\circ} \mathrm{C}$ and spontaneously resolved within 12 hours. The patients with grade 3 and 4 fever were treated with nonsteroidal antiinflammatory drugs, which resolved within 48 hours. No patient exhibited other treatment-associated serious AEs such as infectiousness, vitiligo, nausea, or vomiting.

3.5. Treatment Efficacy. The ORR was observed in 20 (25.0\%) out of 80 patients, in which 4 underwent CR whereas 16, exhibited PR. In addition to the response level, DCR was observed in $50(62.5 \%)$ patients. At the end of follow-up in July 2020, all cancers had progressed significantly in all patients, and 70 patients had died of the disease. The medium time for PFS and OS was 6.1 and 11.3 months, respectively (Figures 1(a) and 1(b)). Notably, the patients who underwent CR were 35 and 50 years old, presenting with lung and liver metastases, respectively. After 12 weeks of the combined therapy, the multiple lung (Figure 2(a)) and liver metastases (Figure 2(b)) disappeared. The PFS was 15.4 months for the first patient, and 10.9 months for the second. The two patients are still alive. The median time for PFS and OS for the 20 patients with CR or PR was 12.8 and 25.8 months, respectively. Of note is that 7 of the 20 patients who underwent PR are still alive and undergoing the last follow-up.

3.6. The ORR and Non-ORR of the Study Cohort. The mPFS and mOS of the patients who displayed ORR $(N=20)$ and patients that did not show ORR $(N=60)$ were 12.8 months vs. 5.3 months $(P<0.0001)$ and 25.8 months vs. 9.1 months 


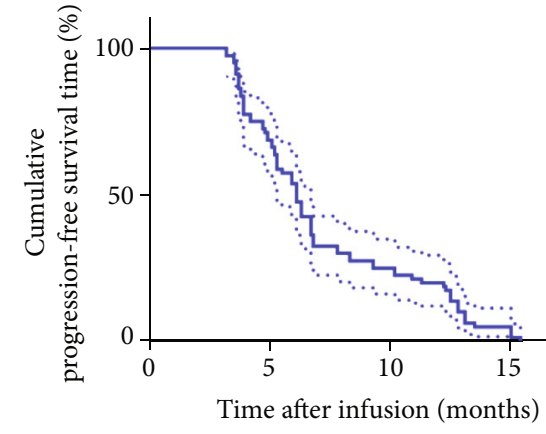

(a)

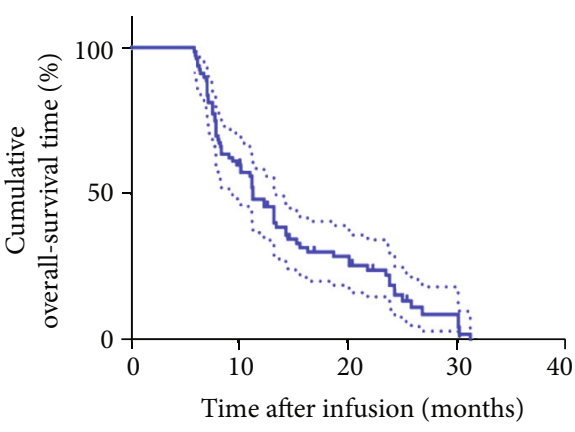

(b)

FIgure 1: Kaplan-Meier curves for PFS and OS of metastatic cervical cancer. (a) Patients' PFS curve. (b) Patients' OS curve.

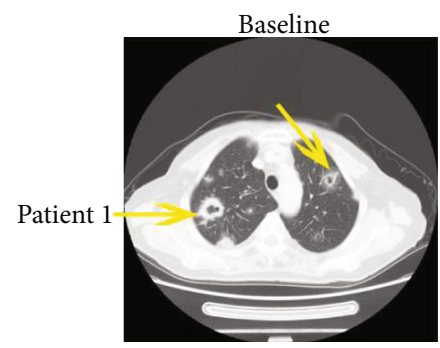

(a)
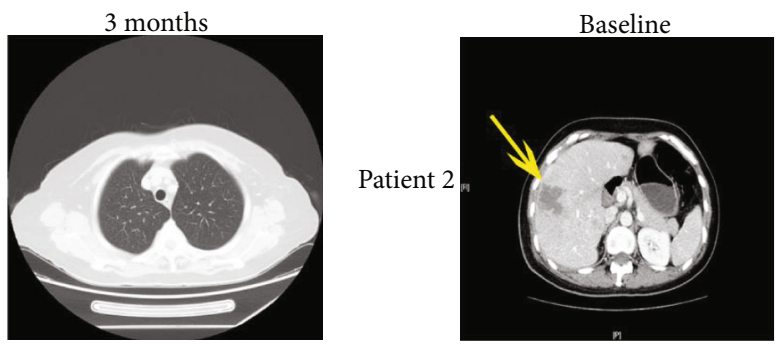

(b)

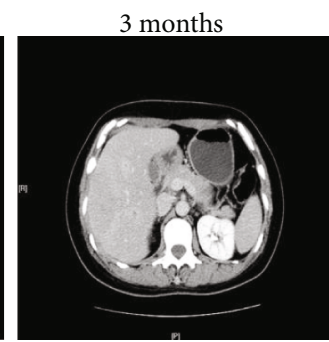

FIGURE 2: Patients exhibiting a CR of multiple lung metastases and liver metastases after TILs plus anti-PD1 therapy. (a) The patient achieved a CR of multiple lung metastases (the yellow arrows) after 3 months of immunotherapy. (b) The patient achieved a CR of multiple liver metastases (the yellow arrows) after 4 months of immunotherapy.

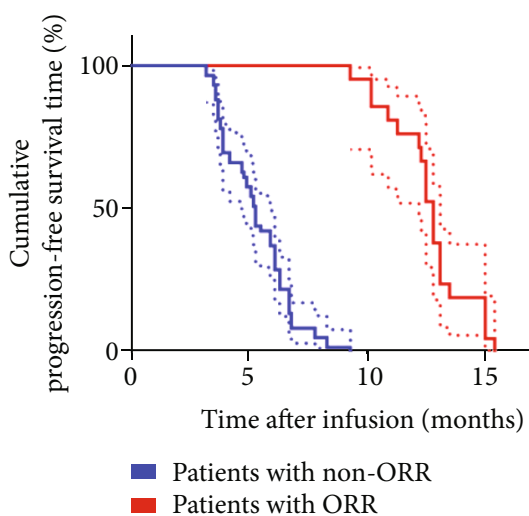

(a)

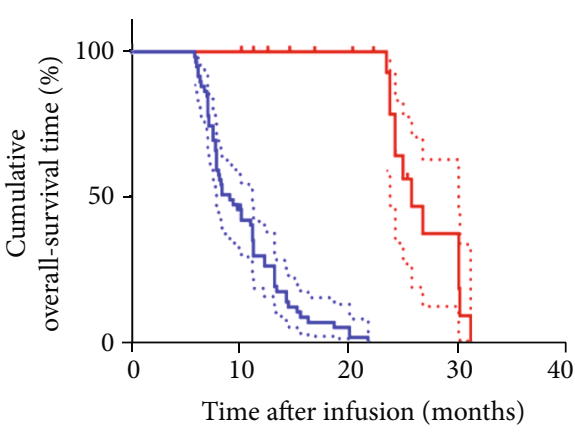

(b)

FIgURE 3: Kaplan-Meier curves for PFS and OS of patients with ORR (CR+PR), $N=20$ and non-ORR, $N=60$. (a) The PFS curve of patients with ORR and non-ORR. (b) The OS curve of patients with ORR and non-ORR.

$(P<0.0001)$, respectively (Figures 3(a) and 3(b)). Notably, patients who exhibited ORR were more likely to show better response to TILs and anti-PD1 combination therapy. Thus, we further explored potential biomarkers that could predict efficacy of this therapy based on the characteristics of patients who exhibited ORR. The first analysis was performed for age, ECOG PS, size of primary tumor, pathological type, type of first-line chemotherapy (with bevacizumab or not), and location of metastatic tumors. Surprisingly, there was no significant difference among these factors between the two groups of patients (ORR and non-ORR) (Table 3). Interestingly, significant differences in the number of infusion TIL; percentage of $\mathrm{CD}^{+} \mathrm{TIL}, \mathrm{CD} 8^{+} \mathrm{PD} 1^{+} \mathrm{TIL}$, and $\mathrm{CD} 4^{+} \mathrm{FoxP}^{+}$ TIL; status of HPV infection; and development of fever after immunotherapy were reported between the two groups of patients (Table 3 ). The number of infused TIL and percentage of $\mathrm{CD}^{+}$TIL in patients who underwent ORR versus those who did not were $50 \times 10^{9} \pm 5.2 \times 10^{9}$ vs. $28 \times 10^{9} \pm$ $2.3 \times 10^{9}(P<0.0001)$ and $76.8 \% \pm 3.5 \%$ vs. $51.3 \% \pm 3.2 \%$ $(P<0.0001)$, respectively. Conversely, percentage of infused $\mathrm{CD} 8^{+} \mathrm{PD} 1^{+}$TIL and $\mathrm{CD} 4^{+} \mathrm{FoxP}^{+}$TIL in patients who exhibited ORR versus patients who did not were $6.2 \% \pm 1.4 \%$ vs. 
TABLE 3: Characteristics of patients with ORR $(N=20)$ and nonORR $(N=60)$.

\begin{tabular}{|c|c|c|c|}
\hline Characteristic & $\begin{array}{l}\text { No. of } \\
\text { ORR }\end{array}$ & $\begin{array}{l}\text { No. of non- } \\
\text { ORR }\end{array}$ & $\begin{array}{c}P \\
\text { value }\end{array}$ \\
\hline \multicolumn{4}{|l|}{ Age (years) } \\
\hline$\geq 45$ & 11 & 34 & \\
\hline$<45$ & 9 & 26 & 0.896 \\
\hline \multicolumn{4}{|l|}{ Pathological type } \\
\hline Squamous cell & 16 & 49 & \\
\hline Adenocarcinoma & 4 & 11 & 0.869 \\
\hline \multicolumn{4}{|l|}{ ECOG PS } \\
\hline 0 & 13 & 37 & \\
\hline 1 & 7 & 23 & 0.790 \\
\hline \multicolumn{4}{|c|}{ Size of primary tumor $(\mathrm{cm})$} \\
\hline$\geq 5$ & 9 & 38 & \\
\hline$<5$ & 11 & 22 & 0.149 \\
\hline \multicolumn{4}{|c|}{$\begin{array}{l}\text { First-line chemotherapy with } \\
\text { bevacizumab }\end{array}$} \\
\hline Yes & 8 & 12 & \\
\hline No & 12 & 48 & 0.074 \\
\hline \multicolumn{4}{|c|}{ Location of metastatic tumors } \\
\hline Lung & 11 & 45 & \\
\hline Others & 9 & 15 & 0.091 \\
\hline \multicolumn{4}{|c|}{ Infusion of TIL numbers } \\
\hline$\geq 50 \times 10^{9}$ & 17 & 10 & \\
\hline$<50 \times 10^{9}$ & 3 & 50 & 0.000 \\
\hline \multicolumn{4}{|c|}{ Infusion of $\mathrm{CD} 8^{+} \mathrm{TIL}$ percentage } \\
\hline$\geq 60 \%$ & 18 & 11 & \\
\hline$<60 \%$ & 2 & 49 & 0.000 \\
\hline \multicolumn{4}{|c|}{$\begin{array}{l}\text { Infusion of } \mathrm{CD}^{+} \mathrm{PD} 1^{+} \mathrm{TIL} \\
\text { percentage }\end{array}$} \\
\hline$\geq 10 \%$ & 3 & 35 & \\
\hline$<10 \%$ & 17 & 25 & 0.001 \\
\hline \multicolumn{4}{|c|}{$\begin{array}{l}\text { Infusion of } \mathrm{CD}^{+}{ }^{+} \mathrm{FoxP}^{+} \mathrm{TIL} \\
\text { percentage }\end{array}$} \\
\hline$\geq 20 \%$ & 4 & 33 & \\
\hline$<20 \%$ & 16 & 27 & 0.007 \\
\hline \multicolumn{4}{|l|}{ HPV status } \\
\hline Positive & 20 & 48 & \\
\hline Negative & 0 & 12 & 0.030 \\
\hline \multicolumn{4}{|l|}{ Fever } \\
\hline Yes & 20 & 34 & \\
\hline No & 0 & 26 & 0.000 \\
\hline
\end{tabular}

$26.3 \% \pm 2.9 \%(P<0.0001)$ and $10.3 \% \pm 1.9 \%$ vs. $25.4 \% \pm$ $5.3 \%(P<0.0001)$, respectively. Additionally, many patients in the ORR group developed fever and tested positive for HPV (100\% vs. $50 \%$ and $100 \%$ vs. $80 \%$ ), respectively. Based on these findings, higher infusion of TIL cells and the percentage of $\mathrm{CD}^{+}$TIL, less infusion of $\mathrm{CD} 8^{+} \mathrm{PD} 1^{+}$TIL and $\mathrm{CD}^{+}{ }^{\mathrm{FoxP}_{3}}{ }^{+} \mathrm{TIL}$, and infection with HPV and fever are potential factors predicting the response to TILs and antiPD1 combination therapy.
TABLE 4: Univariate analysis of factors related to mPFS and mOS of patients in this study $(\mathrm{N}=80)$.

\begin{tabular}{|c|c|c|c|c|}
\hline Characteristics & $\begin{array}{c}\text { mPFS } \\
\text { (months) }\end{array}$ & $\begin{array}{c}P \\
\text { value }\end{array}$ & $\begin{array}{c}\text { mOS } \\
\text { (months) }\end{array}$ & $\begin{array}{c}P \\
\text { value }\end{array}$ \\
\hline \multicolumn{5}{|l|}{ Age (years) } \\
\hline$\geq 45$ & 5.50 & & 10.2 & \\
\hline$<45$ & 6.80 & 0.613 & 13.4 & 0.395 \\
\hline \multicolumn{5}{|l|}{ Pathological type } \\
\hline Squamous cell & 6.10 & & 11.3 & \\
\hline Adenocarcinoma & 3.90 & 0.639 & 7.60 & 0.791 \\
\hline \multicolumn{5}{|l|}{ ECOG PS } \\
\hline 0 & 7.30 & & 13.4 & \\
\hline 1 & 5.90 & 0.562 & 11.2 & 0.823 \\
\hline \multicolumn{5}{|c|}{ Size of primary tumor $(\mathrm{cm})$} \\
\hline$\geq 5$ & 6.70 & & 13.2 & \\
\hline$<5$ & 6.10 & 0.115 & 11.2 & 0.895 \\
\hline \multicolumn{5}{|c|}{$\begin{array}{l}\text { First-line chemotherapy } \\
\text { with bevacizumab }\end{array}$} \\
\hline Yes & 6.30 & & 11.3 & \\
\hline No & 4.45 & 0.400 & 8.00 & 0.157 \\
\hline \multicolumn{5}{|l|}{$\begin{array}{l}\text { Location of metastatic } \\
\text { tumors }\end{array}$} \\
\hline Lung & 8.30 & & 15.3 & \\
\hline Others & 6.10 & 0.931 & 11.3 & 0.130 \\
\hline
\end{tabular}

3.7. Prognostic Factors Associated with TIL and Anti-PD1 Combination Therapy. There was higher infusion of TIL, higher percentage of $\mathrm{CD} 8^{+} \mathrm{TIL}$ but lower infusion of $\mathrm{CD}^{+-}$ $\mathrm{PD}^{+}$and $\mathrm{CD}^{+}{ }^{+} \mathrm{FoxP}^{+}$TIL in patients who displayed ORR. Additionally, patients with ORR were all positive for HPV and developed fever after immunotherapy. Based on this, we assessed potential prognostic factors that may predict clinical response to the combined therapy. Moreover, Kaplan-Meier analysis revealed that no significant difference in medium PFS and OS for different age groups, ECOG PS, size of primary tumor, pathological type, first-line chemotherapy (with bevacizumab or not), and location of metastatic tumors (Table 4). In contrast, univariate analyses revealed that higher infusion of TIL and CD8 ${ }^{+}$TIL percentage, lower infusion of $\mathrm{CD}^{+} \mathrm{PD}^{+}$and $\mathrm{CD}^{+} \mathrm{FoxP}^{+} \mathrm{TIL}$, and infection with HPV and development of fever were significantly associated with higher medium PFS (8.3 months vs. 3.9 months, $P<0.0001 ; 6.8$ months vs. 3.8 months, $P<0.0001 ; 12.5$ months vs. 5.2 months, $P<0.0001$; 11.75 months vs. 4.9 months, $P<0.0001 ; 6.5$ months vs. 3.6 months, $P<0.0001$; and 6.8 months vs. 3.85 months, $P<$ 0.0001) (Figures 4(a) $-4(\mathrm{f})$ ), as well as medium OS (18.7 months vs. 7.6 months, $P<0.0001 ; 15.3$ months vs. 7.1 months, $P<0.0001$; 25.0 months vs. 8.4 months, $P<0.0001$; 23.8 months vs. 8.0 months, $P<0.0001 ; 13.2$ months vs. 6.45 months, $P<0.0001$; and 15.3 months vs. 7.15 months, $P<$ 0.0001 ) (Figures 5(a)-5(f)). Similar findings were obtained in the multivariate Cox proportional hazard model $(P<0.0001)$ for both median PFS (Table 5) and OS (Table 6). In conclusion, higher infusion of TIL and CD8 ${ }^{+}$TIL, lower infusion of 


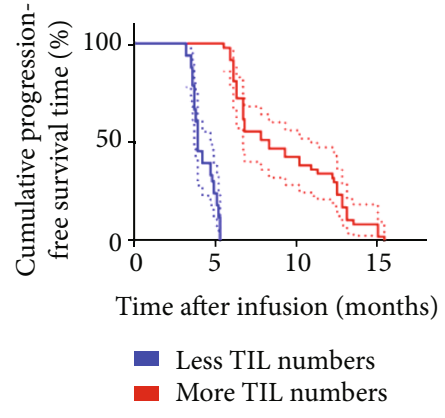

(a)

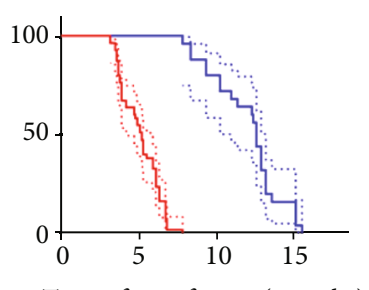

Time after infusion (months)

Less CD8 ${ }^{+} \mathrm{PD} 1^{+} \mathrm{TIL}$ - More $\mathrm{CD} 8^{+} \mathrm{PD} 1^{+} \mathrm{TIL}$

(c)

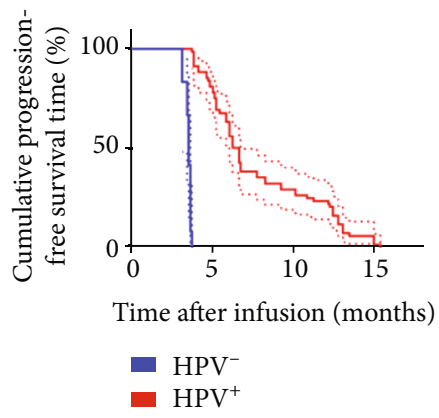

(e)

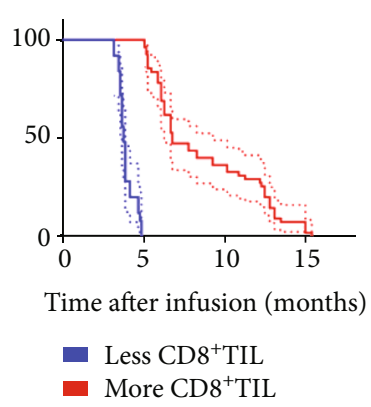

(b)

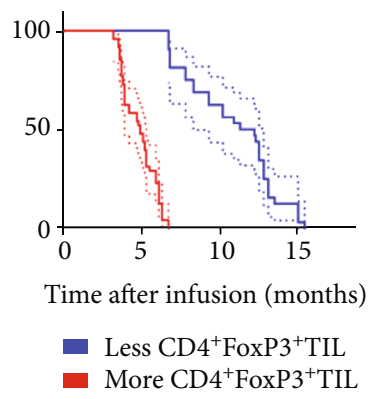

(d)

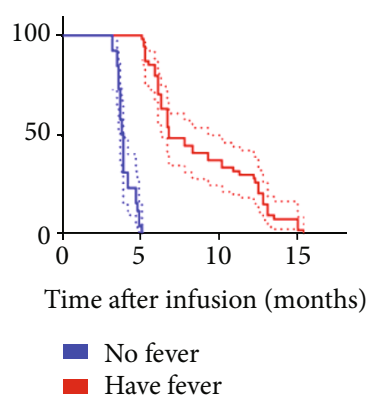

(f)

FIgURE 4: Univariate analyses of more infusion (TIL numbers and CD8 ${ }^{+}$TIL percentage), less infusion (CD8 ${ }^{+} \mathrm{PD} 1^{+} \mathrm{TIL}^{\mathrm{T}}$ percentage and $\mathrm{CD} 4^{+}$FoxP $3^{+}$TIL percentage), and HPV status and having fever or not after immunotherapy based on PFS. PFS curve for: (a) patients with more TIL numbers $\left(\geq 50 \times 10^{9}\right.$, red line) and less TIL numbers $\left(<50 \times 10^{9}\right.$, blue line); (b) patients with more CD $8^{+}$TIL $(\geq 60 \%$, red line) and less $\mathrm{CD}^{+}$TIL ( $<60 \%$, blue line); (c) patients with more $\mathrm{CD}^{+}{ }^{+} \mathrm{PD} 1^{+}$TIL $\left(\geq 10 \%\right.$, red line) and less $\mathrm{CD} 8^{+} \mathrm{PD} 1^{+} \mathrm{TIL}(<10 \%$, blue line); (d) patients with more $\mathrm{CD}^{+}{ }^{+} \mathrm{FoxP}^{+}$TIL $\left(\geq 20 \%\right.$, red line) and less $\mathrm{CD}^{+} \mathrm{FoxP}^{+}$TIL $\left(<20 \%\right.$, blue line); (e) patients with $\mathrm{HPV}^{+}$(red line) and $\mathrm{HPV}^{-}$(blue line); and (f) patients having fever (red line) and no fever (blue line) after immunotherapy.

$\mathrm{CD} 8^{+} \mathrm{PD} 1^{+} \mathrm{TIL}$ and $\mathrm{CD} 4^{+} \mathrm{FoxP}^{+} \mathrm{TIL}$, and infection with HPV and occurrence of fever are potential factors for predicting the clinical response to combination therapy of TILs and anti-PD1.

\section{Discussion}

Although many clinical studies have demonstrated longterm survival benefits of immunotherapy in various cancer entities, little is known whether this therapy is effective against cervical cancers. In the United States, pembrolizumab, an antibody against $1 \mathrm{PD}-1$, is approved for advanced endometrial cancers with high levels of microsatellite instability (MSI-high) and for recurrent or progressive metastatic cervical cancer positive for programmed death-ligand 1 (PD-L1). This suggests that only a specific group of patients benefit from this immunotherapy [27]. Patients with meta- static human papillomavirus (HPV)-associated carcinomas put on TILs therapy have shown modest response to the treatment [19]. However, effective immunotherapies for the treatment of metastatic cervical cancers accompanied with low MSI expression and negative for PDL1 are limited. Here, we explored the efficacy of adoptive transfer of TILs and anti-PD1 antibody in patients with metastatic cervical cancer showing low MSI expression and negative for PDL1. Interestingly, we found that this combination therapy registered promising antitumor effects and a satisfactory objective response, with clinical tumor regression observed in 20 out of the 80 patients (25.0\%). These findings suggest that a combination therapy of TILs and anti-PD1 may potentially modulate the growth of metastatic cervical cancers in patients with low MSI expression and negative for PDL1. 


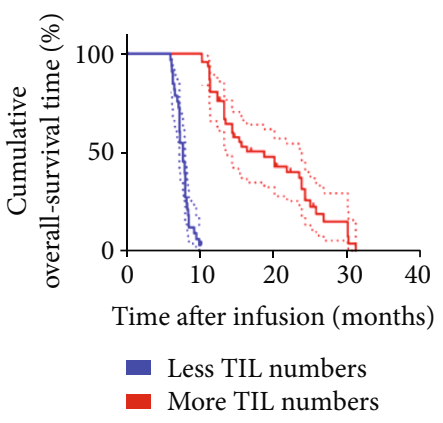

(a)

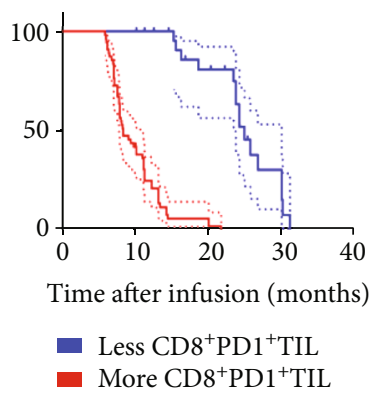

(c)

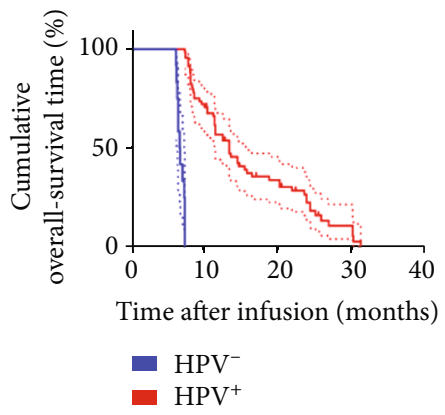

(e)

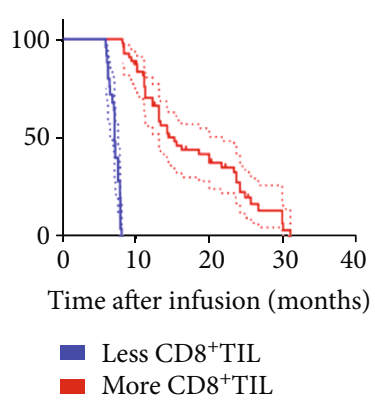

(b)

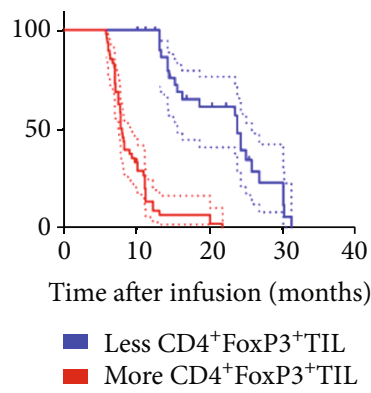

(d)

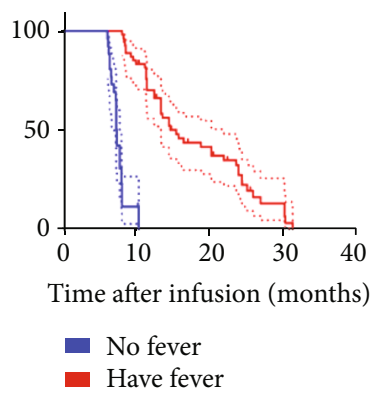

(f)

FIgURE 5: Univariate analyses of more infusion (TIL numbers and CD8 ${ }^{+}$TIL percentage), less infusion (CD8 ${ }^{+} \mathrm{PD} 1^{+}$TIL percentage and $\mathrm{CD}^{+}{ }^{+} \mathrm{FoxP}^{+}$TIL percentage), and HPV status and having fever or not after immunotherapy based on OS. OS curve for: (a) patients with more TIL numbers $\left(\geq 50 \times 10^{9}\right.$, red line) and less TIL numbers $\left(<50 \times 10^{9}\right.$, blue line); (b) patients with more CD $8^{+}$TIL ( $\geq 60 \%$, red line) and less $\mathrm{CD}^{+}$TIL ( $<60 \%$, blue line); (c) patients with more CD8 ${ }^{+} \mathrm{PD} 1^{+}$TIL $\left(\geq 10 \%\right.$, red line) and less $\mathrm{CD} 8^{+} \mathrm{PD} 1^{+} \mathrm{TIL}(<10 \%$, blue line); (d) patients with more $\mathrm{CD}^{+} \mathrm{FoxP}^{+}$TIL $\left(\geq 20 \%\right.$, red line) and less $\mathrm{CD}^{+} \mathrm{FoxP}^{+}$TIL $\left(<20 \%\right.$, blue line); (e) patients with $\mathrm{HPV}^{+}$(red line) and $\mathrm{HPV}^{-}$(blue line); and (f) patients having fever (red line) and no fever (blue line) after immunotherapy.

TABLE 5: Multivariate analysis (mPFS).

\begin{tabular}{|c|c|c|c|}
\hline Parameters & Hazard ratio & $95 \%$ confidence interval & $P$ value \\
\hline Infusion of $\mathrm{CD}^{+}$TIL numbers $\left(\geq 50 \times 10^{9}\right.$ vs. $\left.<50 \times 10^{9}\right)$ & 5.823 & $(2.871,11.81)$ & $<0.0001$ \\
\hline Infusion of $\mathrm{CD}^{+}$TIL percentage ( $\geq 60 \%$ vs. $<60 \%$ ) & 7.05 & $(2.813,17.67)$ & $<0.0001$ \\
\hline Infusion of $\mathrm{CD}^{+} \mathrm{PD}^{+}$TIL percentage $(\geq 10 \%$ vs. $<10 \%)$ & 4.285 & $(2.697,6.807)$ & $<0.0001$ \\
\hline Infusion of $\mathrm{CD}^{+} \mathrm{FoxP}^{+}{ }^{+}$TIL percentage ( $\geq 20 \%$ vs. $\left.<20 \%\right)$ & 4.551 & $(2.74,7.561)$ & $<0.0001$ \\
\hline HPV status (positive vs. negative) & 11.84 & $(1.915,73.16)$ & $<0.0001$ \\
\hline Fever (yes vs. no) & 6.857 & $(2.83,16.62)$ & $<0.0001$ \\
\hline
\end{tabular}

This research was motivated by the lack of effective treatment options for metastatic cervical cancers, particularly in patients who fail to respond to first-line therapy. Out of the 80 patients, 20 showed objective response, in which 4 underwent CR, whereas 16 exhibited PR. The medium PFS and OS were 6.1 and 11.3 months, respectively. Previous studies (KEYNOTE-028 and KEYNOTE-158) have reported considerable benefits of anti-PD1 therapy in controlling persistent and advanced cervical cancer $[28,29]$. In these studies, however, patients with lacking PD-L1 expression did not respond 
TABLe 6: Multivariate analysis (mOS).

\begin{tabular}{|c|c|c|c|}
\hline Parameters & Hazard ratio & $95 \%$ confidence interval & $P$ value \\
\hline Infusion of CD8 ${ }^{+}$TIL numbers $\left(\geq 50 \times 10^{9}\right.$ vs. $\left.<50 \times 10^{9}\right)$ & 6.169 & $(3.001,12.68)$ & $<0.0001$ \\
\hline Infusion of $\mathrm{CD} 8^{+}$TIL percentage ( $\geq 60 \%$ vs. $\left.<60 \%\right)$ & 7.569 & $(2.992,19.15)$ & $<0.0001$ \\
\hline Infusion of $\mathrm{CD}^{+} \mathrm{PD}^{+}$TIL percentage ( $\geq 10 \%$ vs. $\left.<10 \%\right)$ & 5.321 & $(3.29,8.606)$ & $<0.0001$ \\
\hline Infusion of $\mathrm{CD}^{+}{ }^{+} \mathrm{FoxP}^{+}{ }^{+}$TIL percentage ( $\geq 20 \%$ vs. $<20 \%$ ) & 4.916 & $(2.939,8.222)$ & $<0.0001$ \\
\hline HPV status (positive vs. negative) & 12.74 & $(2.967,82.5)$ & $<0.0001$ \\
\hline Fever (yes vs. no) & 6.314 & $(2.674,14.91)$ & $<0.0001$ \\
\hline
\end{tabular}

to immunotherapy. Despite being a single-center, single-arm retrospective clinical study, our findings suggest that a combination therapy of TILs and anti-PD1 may be effective against metastatic cervical cancers resistant to chemotherapy, and in patients with low MSI expression and PDL1-negative.

The effects of anti-PD1 therapy are mediated by TILs in the tumor microenvironment; therefore, combining antiPD1 with TIL therapy may confer more effective antitumor effects on metastatic cervical cancer. Previous studies have shown that IFN- $\gamma$, an inflammatory cytokine, produced by TILs induces expression of PD-L1 in tumor cells [30]. Although, we did explore the mechanism underlying ORR in the 20 patients who showed positive response to the therapy; we speculate that TILs penetrate the tumor microenvironment and secrete IFN- $\gamma$, which induces expression of PD-L1 in the tumor cells. Cancer cells expressing this ligand are thus recognized, bound by PD1 antibodies, and eventually killed by Ab-mediated processes. Further studies should be conducted to explore the effect of adoptive transfer of TILs in ablating tumors when combined with anti-PD1 therapy.

The most common treatment-related adverse events of any grade were fever, fatigue, rash, and anorexia. There were few AEs of grade 3 or 4 , especially when considered in the context of the cytotoxic chemotherapy agents that patients in this population had previously received. Four patients who exhibited grade 3 or 4 fever were effectively treated with nonsteroidal anti-inflammatory drugs and resolved within 48 hours. Interestingly, two of the four patients underwent CR. Univariate and multivariate analyses showed that patients who developed fever after immunotherapy exhibit longer PFS and higher OS. In one study, it was found that fever after anti-PD1 therapy may be an early predictor for better response to anti-PD1 treatment; this is in agreement with our findings. However, future studies should focus on exploring the association between fever and immunotherapy.

Persistent HPV infection influences the pathogenesis and prognosis of cervical cancer. Whereas HPV-associated cancers constitutively express HPV E6 and E7 oncoproteins; immunologically foreign viral proteins that are attractive targets for immunotherapy [31], targeting HPV oncoproteins with antigen-specific immunotherapy using therapeutic vaccines has so far been ineffective for metastatic disease [19]. One study suggested that the association between HPV status with the efficacy of PD-1/PD-L1 inhibitors against cervical cancer is not clear due to the paucity of available data [16]. Nonetheless, cultured TILs have been shown to mediate the regression of HPV-associated epithelial cancers including cervical cancer $[19,20]$. Moreover, the reactivity of HPV with the infused T cells together with repopulation of peripheral blood with HPV-reactive $\mathrm{T}$ cells also correlated with positive clinical response [19]. In this study, univariate and multivariate analyses showed that patients not infected with HPV exhibited better PFS and OS. Based on this finding, HPV status may predict the prognosis of patients following immunotherapy.

Consistent with previous findings $[23,24]$, univariate and multivariate analyses indicated that patients with higher infusion of TILs and CD8 ${ }^{+}$TILs exhibited better PFS and OS. Conversely, higher infusion of $\mathrm{CD} 8^{+} \mathrm{PD} 1^{+} \mathrm{TIL}$ and $\mathrm{CD} 4^{+-}$ $\mathrm{FoxP}^{+}$TIL resulted in poor PFS and OS. Expression of PD1 by TILs is thought to be one of the factors that weaken antitumor immune response [32]. Consistently, individuals with higher infusion of $\mathrm{CD} 88^{+} \mathrm{PD} 1^{+}$TIL exhibited poor PFS and low OS. One recent study reported that blocking the PD-1 pathway significantly increased antitumor effects of adoptive immunotherapy performed using $\mathrm{T}$ cells with chimeric antigen receptor (CAR) [33]. In this study, a subpopulation of PD $-1^{+} \mathrm{T}$ lymphocytes was observed in the cultured TILs, suggesting that a PD-1 blockade may significantly increase the cytotoxicity of TILs. Regulatory T cells (Tregs) have been shown to suppress $\mathrm{T}$ cell-mediated host immune response against self- and nonself-antigens [34-36]. In fact, studies have described a negative relationship between peripheral $\mathrm{CD} 4^{+} \mathrm{FoxP} 3^{+}$regulatory $\mathrm{T}$ cell levels and clinical response to adoptive immunotherapy of human cancer [37], suggesting that Tregs mediate the inhibitory effects of adoptive immunotherapy on human tumors. In this study, we found that higher infusion of $\mathrm{CD}^{+} \mathrm{FoxP}^{+}$TIL resulted in poor PFS and OS, suggesting that eliminating the suppressive T-regulatory lymphocytes can enhance antitumor efficacy.

This study was carried out in a single-center, with singlearm and retrospective clinical design. However, we show that a combination therapy of TILs and anti-PD1 may potentially inhibit the growth of metastatic cervical cancers in patients with low MSI expression and negative for PDL1. However, whether TILs should be administered in combination with anti-PD-1 or as a single treatment option in patients with metastatic cervical cancer is still unclear. Further studies should be conducted to clarify this. Moreover, the reliability of using cultured TILs as biomarkers for predicting clinical response to the combination immunotherapy should be further investigated.

\section{Conclusion}

In conclusion, our results show that a combination therapy of TILs with anti-PD1 presents an effective approach for 
controlling metastatic cervical cancer in patients with low MSI-1 and PDL1 negative. However, further studies are needed to validate these findings and describe the specific mechanisms underlying the use of TILs anti-PD1 therapy.

\section{Data Availability}

All data generated in the study are included in the present article.

\section{Ethical Approval}

All procedures performed were in accordance with the ethical standards of the institutional and/or national Research committee and with the 1964 Helsinki declaration and its later amendments or comparable ethical standards.

\section{Consent}

Institutional review board approval and data sharing agreements were obtained from all participating institutions. All data were anonymized.

\section{Conflicts of Interest}

The authors declare that they have no competing interests.

\section{Acknowledgments}

We thank all the enrolled patients and their cooperation. We thank all the staff of GMP lab for the preparation of TILs.

\section{Supplementary Materials}

Supplementary 1. Supplementary Figure 1: representative flow cytometry of $\mathrm{CD}^{+}, \mathrm{CD}^{+} \mathrm{CD}^{+}, \mathrm{CD}^{+} \mathrm{CD}^{+}, \mathrm{CD}^{+-}$ $\mathrm{CD}_{56}{ }^{+}, \mathrm{CD}^{-} \mathrm{CD}_{56}{ }^{+}, \mathrm{CD}^{+} \mathrm{CD}^{+} \mathrm{PD}^{+}, \mathrm{CD} 4^{+} \mathrm{FoxP}^{+}$percentage of TILs.

Supplementary 2. Supplementary Figure 2: cytotoxicity and cytokines production by TILs. (A) The cytotoxicity of TILs against Hela target cell line; (B) representative flow cytometry of IFN- $\gamma$, IL-2, and TNF- $\alpha$ expression on $\mathrm{CD}^{+}$fresh TILs from biopsy samples and cultured TILs. (C) Quantitative analysis of IFN- $\gamma$, IL- 2 , and TNF- $\alpha$ expression on $\mathrm{CD}^{+}$fresh TILs from biopsy samples and cultured TILs.

\section{References}

[1] F. Kamangar, G. M. Dores, and W. F. Anderson, "Patterns of cancer incidence, mortality, and prevalence across five continents: defining priorities to reduce cancer disparities in different geographic regions of the world," Journal of Clinical Oncology, vol. 24, no. 14, pp. 2137-2150, 2006.

[2] A. Jemal, F. Bray, M. M. Center, J. Ferlay, E. Ward, and D. Forman, "Global cancer statistics," CA: a Cancer Journal for Clinicians, vol. 61, no. 2, pp. 69-90, 2011.

[3] R. L. Siegel, K. D. Miller, and A. Jemal, "Cancer statistics, 2020," CA: a Cancer Journal for Clinicians, vol. 70, no. 1, pp. 7-30, 2020.
[4] M. E. Sherman, S. S. Wang, J. Carreon, and S. S. Devesa, "Mortality trends for cervical squamous and adenocarcinoma in the United States: relation to incidence and survival," Cancer, vol. 103, no. 6, pp. 1258-1264, 2005.

[5] H. L. Howe, X. Wu, L. A. G. Ries et al., "Annual report to the nation on the status of cancer, 1975-2003, featuring cancer among U.S. Hispanic/Latino populations," Cancer, vol. 107, no. 8, pp. 1711-1742, 2006.

[6] J. Barnholtz-Sloan, N. Patel, D. Rollison, K. Kortepeter, J. MacKinnon, and A. Giuliano, "Incidence trends of invasive cervical cancer in the United States by combined race and ethnicity," Cancer Causes \& Control, vol. 20, no. 7, pp. 11291138, 2009.

[7] S. S. Wang, J. D. Carreon, S. L. Gomez, and S. S. Devesa, "Cervical cancer incidence among 6 Asian ethnic groups in the United States, 1996 through 2004," Cancer, vol. 116, no. 4, pp. 949-956, 2010.

[8] S. Gupta, A. Maheshwari, P. Parab et al., "Neoadjuvant chemotherapy followed by radical surgery versus concomitant chemotherapy and radiotherapy in patients with stage IB2, IIA, or IIB squamous cervical cancer: a randomized controlled trial," Journal of Clinical Oncology, vol. 36, no. 16, pp. 15481555, 2018.

[9] D. H. Moore, J. A. Blessing, R. P. McQuellon et al., "Phase III study of cisplatin with or without paclitaxel in stage IVB, recurrent, or persistent squamous cell carcinoma of the cervix: a Gynecologic Oncology Group study," Journal of Clinical Oncology, vol. 22, no. 15, pp. 3113-3119, 2004.

[10] H. J. Long III, B. N. Bundy, E. C. Grendys Jr. et al., "Randomized phase III trial of cisplatin with or without topotecan in carcinoma of the uterine cervix: a Gynecologic Oncology Group study," Journal of Clinical Oncology, vol. 23, no. 21, pp. 4626-4633, 2005.

[11] B. J. Monk, M. W. Sill, D. S. McMeekin et al., "Phase III trial of four cisplatin-containing doublet combinations in stage IVB, recurrent, or persistent cervical carcinoma: a Gynecologic Oncology Group study," Journal of Clinical Oncology, vol. 27, no. 28, pp. 4649-4655, 2009.

[12] K. S. Tewari, M. W. Sill, R. T. Penson et al., "Bevacizumab for advanced cervical cancer: final overall survival and adverse event analysis of a randomised, controlled, open-label, phase 3 trial (Gynecologic Oncology Group 240)," Lancet, vol. 390, no. 10103, pp. 1654-1663, 2017.

[13] V. Heong, N. Ngoi, and D. S. P. Tan, "Update on immune checkpoint inhibitors in gynecological cancers," Journal of Gynecologic Oncology, vol. 28, no. 2, article e20, 2017.

[14] P. C. Tumeh, C. L. Harview, J. H. Yearley et al., "PD-1 blockade induces responses by inhibiting adaptive immune resistance," Nature, vol. 515, no. 7528, pp. 568-571, 2014.

[15] L. E. Minion and K. S. Tewari, "Cervical cancer - state of the science: from angiogenesis blockade to checkpoint inhibition," Gynecologic Oncology, vol. 148, no. 3, pp. 609-621, 2018.

[16] Y. Liu, L. Wu, R. Tong et al., "PD-1/PD-L1 inhibitors in cervical cancer," Frontiers in Pharmacology, vol. 10, p. 65, 2019.

[17] A. M. Goodman, S. Kato, L. Bazhenova et al., "Tumor mutational burden as an independent predictor of response to immunotherapy in diverse cancers," Molecular Cancer Therapeutics, vol. 16, no. 11, pp. 2598-2608, 2017.

[18] C. S. Hinrichs, "Molecular pathways: breaking the epithelial cancer barrier for chimeric antigen receptor and t-cell receptor 
gene therapy," Clinical Cancer Research, vol. 22, no. 7, pp. 1559-1564, 2016.

[19] S. Stevanović, S. R. Helman, J. R. Wunderlich et al., "A phase II study of tumor-infiltrating lymphocyte therapy for human papillomavirus-associated epithelial cancers," Clinical Cancer Research, vol. 25, no. 5, pp. 1486-1493, 2019.

[20] S. Stevanović, L. M. Draper, M. M. Langhan et al., "Complete regression of metastatic cervical cancer after treatment with human papillomavirus-targeted tumor-infiltrating T cells," Journal of Clinical Oncology, vol. 33, no. 14, pp. 1543-1550, 2015.

[21] L. H. Schwartz, S. Litière, E. de Vries et al., "RECIST 1.1update and clarification: from the RECIST committee," European Journal of Cancer, vol. 62, pp. 132-137, 2016.

[22] G. T. Jun, J. Ward, and P. J. Clarkson, "Systems modelling approaches to the design of safe healthcare delivery: ease of use and usefulness perceived by healthcare workers," Ergonomics, vol. 53, no. 7, pp. 829-847, 2010.

[23] W. Li, L. Xu, Y. Wang, L. Zhao, D. B. Kellner, and Q. Gao, "Efficacy of tumor-infiltrating lymphocytes combined with IFN- $\alpha$ in Chinese resected stage III malignant melanoma," Journal of Immunology Research, vol. 2017, Article ID 1092507, 8 pages, 2017.

[24] J. Shi, M. Li, and R. Yang, "Tumor-infiltrating lymphocytes as a feasible adjuvant immunotherapy for osteosarcoma with a poor response to neoadjuvant chemotherapy," Immunotherapy, vol. 12, no. 9, pp. 641-652, 2020.

[25] W. Li, Y. Wang, H. Zhao et al., "Identification and transcriptome analysis of erythroblastic island macrophages," Blood, vol. 134, no. 5, pp. 480-491, 2019.

[26] M. Saint-Jean, A. C. Knol, C. Volteau et al., “Adoptive cell therapy with tumor-infiltrating lymphocytes in advanced melanoma patients," Journal of Immunology Research, vol. 2018, Article ID 3530148, 10 pages, 2018.

[27] F. Baettig, T. Vlajnic, M. Vetter et al., "Nivolumab in chemotherapy-resistant cervical cancer: report of a vulvitis as a novel immune-related adverse event and molecular analysis of a persistent complete response," Journal for Immunotherapy of Cancer, vol. 7, no. 1, p. 281, 2019.

[28] H. C. Chung, W. Ros, J. P. Delord et al., "Efficacy and safety of pembrolizumab in previously treated advanced cervical cancer: results from the phase II KEYNOTE-158 study," Journal of Clinical Oncology, vol. 37, no. 17, pp. 1470-1478, 2019.

[29] J. S. Frenel, C. le Tourneau, B. O’Neil et al., “Safety and efficacy of pembrolizumab in advanced, programmed death ligand 1positive cervical cancer: results from the phase IB KEYNOTE-028 trial," Journal of Clinical Oncology, vol. 35, no. 36, pp. 4035-4041, 2017.

[30] M. Mandai, J. Hamanishi, K. Abiko, N. Matsumura, T. Baba, and I. Konishi, "Dual faces of IFN $\gamma$ in cancer progression: a role of pd-11 induction in the determination of pro-and antitumor immunity," Clinical Cancer Research, vol. 22, no. 10, pp. 2329-2334, 2016.

[31] C. L. Trimble and I. H. Frazer, "Development of therapeutic HPV vaccines," The Lancet Oncology, vol. 10, no. 10, pp. 975-980, 2009.

[32] M. Ahmadzadeh, L. A. Johnson, B. Heemskerk et al., “Tumor antigen-specific CD8 T cells infiltrating the tumor express high levels of PD-1 and are functionally impaired," Blood, vol. 114, no. 8, pp. 1537-1544, 2009.
[33] E. A. Chong, J. J. Melenhorst, S. F. Lacey et al., "PD-1 blockade modulates chimeric antigen receptor (CAR)-modified T cells: refueling the CAR," Blood, vol. 129, no. 8, pp. 1039-1041, 2017.

[34] E. M. Shevach, "Mechanisms of foxp 3+ T regulatory cellmediated suppression," Immunity, vol. 30, no. 5, pp. 636645, 2009.

[35] K. Wing and S. Sakaguchi, "Regulatory T cells exert checks and balances on self tolerance and autoimmunity," Nature Immunology, vol. 11, no. 1, pp. 7-13, 2010.

[36] S. Sakaguchi, M. Miyara, C. M. Costantino, and D. A. Hafler, "FOXP3 + regulatory T cells in the human immune system," Nature Reviews. Immunology, vol. 10, no. 7, pp. 490-500, 2010.

[37] X. Yao, M. Ahmadzadeh, Y. C. Lu et al., "Levels of peripheral $\mathrm{CD} 4+$ FoxP3+ regulatory $\mathrm{T}$ cells are negatively associated with clinical response to adoptive immunotherapy of human cancer," Blood, vol. 119, no. 24, pp. 5688-5696, 2012. 